\title{
Displacement mechanism of polymeric surfactant in chemical cold flooding for heavy oil based on microscopic visualization experiments
}

\author{
Fei $\mathrm{Xu}^{1}$, Qiang $\mathrm{Chen}^{2}$, Mengqi $\mathrm{Ma}^{1}$, Yicheng Wang ${ }^{1}$, Fuwei $\mathrm{Yu}^{1}$, Junjian $\mathrm{Li}^{1 \oplus *}$ \\ ${ }^{1}$ State Key Laboratory of Petroleum Resources and Prospecting, China University of Petroleum, Beijing 102249, P. R. China \\ ${ }^{2}$ Aktobe Project Department, CNPC XiBu Drilling Engineering Company Limited, Karamay 830026, P. R. China
}

Keywords:

Polymeric surfactant

microscopic visualization experiments

heavy oil displacement mechanism

chemical cold flooding

Cited as:

Xu, F., Chen, Q., Ma, M., Wang, Y., Yu, F., Li, J. Displacement mechanism of polymeric surfactant in chemical cold flooding for heavy oil based on microscopic visualization experiments. Advances in Geo-Energy Research, 2020, 4(1): 77-85,

doi: 10.26804/ager.2020.01.07.

\begin{abstract}
:
In order to study the microscopic oil displacement mechanism of polymeric surfactant in chemical cold flooding for heavy oil, the indoor microscopic visualization displacement experiments were carried out. The flooding experiment of heavy oil was conducted by using water, osmotic modified oil displacing agent (a kind of polymeric surfactant) and water-in-oil emulsion (obtained by mixing polymeric surfactant and heavy oil) as displacing phases to study the mechanism of polymeric surfactant to enhance oil recovery in heavy oil reservoir. The experimental results show that the polymeric surfactant can increase the viscosity of the water phase, reduce the water-oil mobility ratio, expand the swept area, and there is no obvious fingering phenomenon which occurs during water flooding. The polymeric surfactant has the surfactant characteristics which can reduce the interfacial tension between oil and water to promote the formation of oil droplets with smaller droplet diameter. And the interfacial film composed of polymeric surfactant molecules will be formed on the surface of oil droplets to prevent the coalescence of oil droplets and improve the flow ability of oil phase. The water-in-oil emulsion can be miscible with the oil in heavy oil displacement process, and thus sweeps the areas such as the dead pores which cannot be swept by water and polymeric surfactant flooding, which increases the sweep efficiency to a certain extent.
\end{abstract}

\section{Introduction}

Chemical cold flooding technology is one of the effective technologies for oil extraction, mainly using surfactants, polymers, alkalis and polymeric surfactants (Alvarado and Manrique, 2010). In heavy oil extraction, chemical cold flooding technology could have good effect on reducing oil viscosity, interfacial tension (IFT), mobility ratio and expanding swept area, which is helpful to increase production and decrease oil extraction cost (Gbadamosi et al., 2019).

The polymer flooding can improve the recovery of heavy oil by mainly increasing the viscosity of displacement phase and reducing the mobility ratio (Deng et al., 2014), otherwise increasing oil displacement efficiency due to viscoelasticity (Zhang et al., 2011). Surfactant flooding and alkali flooding mainly improve oil displacement efficiency by reducing the surface tension between oil and water interfaces, and most researchers believe that the improvement of heavy oil recovery is mainly due to the formation of emulsion, including oilin-water emulsion and water-in-oil emulsion (Shang et al., 2019; Yu et al., 2019). The oil-in-water emulsion with low viscosity was obtained by mixing a viscosity-reducing system composed of surfactants with heavy oil (Lee and Babadagli, 2018), and the water-in-oil emulsion was also obtained by alkali flooding heavy oil (Dranchuk et al., 1974; Faroijq et al., 1979), and both of those oil recovery are better than that of water flooding. Polymeric surfactant is a new type of oil-displacing agent based on polymer flooding and alkaline/surfactant/polymer (ASP) flooding in recent years, having dual properties of both polymer and surfactant with obvious emulsifying, tackification and solubilization ability, which cannot only enlarge the swept volume but also improve

*Corresponding author.

E-mail address: xufei960200@163.com (F. Xu); 630012696@qq.com (Q. Chen);13691252916@163.com (M. Ma);

yicheng751@gmail.com (Y. Wang); 2017312045@student.cup.edu.cn (F. Yu); junjian@cup.edu.cn (J. Li).

2207-9963 (c) The Author(s) 2020.

Received February 18, 2020; revised March 1, 2020; accepted March 2, 2020; available online March 4, 2020. 
the efficiency of oil displacement. And the results of laboratory experiments show that the oil recovery of polymeric surfactant flooding can be improved by more than $15 \%$ compared with water flooding (Sun et al., 2010; Yan et al., 2014). Compared with ordinary polymer, the greatest features of polymeric surfactant are its low concentration with high efficiency, lower IFT and its strong ability to increase viscosity, solubilization and crude oil emulsion (Cao and $\mathrm{Li}, 2002$; $\mathrm{Li}$ et al., 2012; $\mathrm{Wu}$ et al., 2018). Thus, it can be seen that the polymeric surfactant has a good oil recovery effect for its characteristics of high viscosity and reducing viscosity of crude oil, hence it might also have good applicability to the high viscosity heavy oil reservoir. However, the use of polymeric surfactant for heavy oil displacement is relatively few, and the micromechanism of polymeric surfactant displacing heavy oil is still unclear, therefore experiments are needed to study the displacement effect and mechanism of polymeric surfactant flooding heavy oil. In order to make clear the function of polymeric surfactant in chemical cold flooding for heavy oil, this paper uses the microfluidic model to carry out the indoor microscopic visualization displacement experiments.

Microfluidics is a scientific discipline that studies movement, handling and manipulation of liquid substances in microvolumes across nano-, micro- and macro-scales. This ability to precisely manipulate liquids in such small volumes comes from the use of devices with features on micro- and nanoscale where capillary forces and surface tension overcome the effects of gravity and inertia and become dominant forces that enable such control (Lifton, 2016). Thus, microfluidics is able to manipulate fluid in micron-scale space, reducing the basic functions of biological, chemical, and other laboratories to few square centimeters of models (Wang et al., 2019). Nowadays, microfluidic technology has been widely used in aerospace, biochemistry, medical diagnosis, environmental detection and other fields. And this technology has been more and more applied to the flow visualization research of fluid in porous media, which has the characteristics of small sample consumption, high degree of automation and fast analysis speed (Li et al., 2018). At present, many researchers have used microfluidic technology to study the heavy oil displacement, such as Wang (Wang et al., 2014) has used homogeneous and heterogeneous microfluidic models to study the heavy oil gravity drainage, Jamshidi (Jamshidi et al., 2018) has studied the performance of carbonated water injection (CWI) and found that CWI could be a viable option for enhanced oil recovery in heavy oil reservoirs, Mohammadzadeh (Mohammadzadeh et al., 2019) has studied the chemical-assisted waterflooding effect by using visual microscopic model, but there is still few study on polymeric surfactant flooding heavy oil. Therefore, it can be seen that the mechanism of polymeric surfactant in chemical cold flooding for heavy oil can be studied by using microfluidic technology, and it is of significance and value to carry out microscopic experiment of polymeric surfactant flooding heavy oil.

In this paper, experiments on microscopic displacement of heavy oil are carried out by using water, polymeric surfactant and water-in-oil emulsion (obtained by mixing this agent with heavy oil) to displace heavy oil, which aims to study the en-

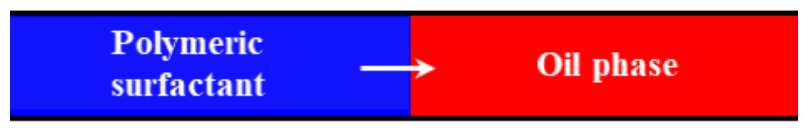

(a)

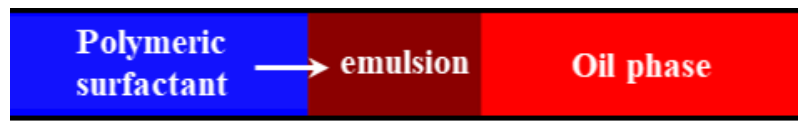

(b)

Fig. 1. Two forms of displacement.

hanced oil recovery (EOR) mechanism of polymeric surfactant. The oil-in-water experiment is left out due to its similar effects as polymeric surfactant flooding.

\section{Materials and methods}

\subsection{Materials}

The oil used in the experiment is the heavy oil (density: $0.916 \mathrm{~g} / \mathrm{cm}^{3}$; viscosity: $173.5 \mathrm{mPa} \cdot \mathrm{s}$ ) obtained by mixing kerosene and heavy oil from Shengli Gudao oil field. The water used in the experiment is deionized water. And the chemical EOR agent used in the experiment is osmotic modified oil displacing agent, a kind of polymeric surfactant (concentration: $0.1 \mathrm{wt} \%$; viscosity: $60 \mathrm{mPa} \cdot \mathrm{s}$; surface tension: $0.024 \mathrm{~N} / \mathrm{m}$; contact angle: $45.1 \sim 50.0^{\circ}$ ). The emulsion used in the experiment is water-in-oil emulsion obtained by mixing polymeric surfactant agent and heavy oil at 1:1 ratio.

\subsection{Experimental methods and principles}

In this paper, the microfluidic glass model is used to visualize the process of heavy oil displacement, collect and analyze the image of the heavy oil displacement process.

The polymeric surfactant has the dual flooding oil characteristics of polymers and surfactants (Raffa et al., 2016), which not only has the effect of polymer flooding to expand the swept area, but also the effect of surfactant flooding to emulsify heavy oil. Therefore, in the process of displacing heavy oil, as shown in Fig. 1, there are two forms of displacement: One is the direct displacement for heavy oil through polymeric surfactant flooding. In the process, the oil-in-water emulsion is formed in partial displacing front and continues to displace heavy oil. Due to the low viscosity of oil-in-water emulsion and the polymeric surfactant agent existing as the continuous phase, the effect of the oil-in-water flooding is similar to that of the polymeric surfactant agent flooding. Another one is that the water-in-oil emulsion is formed in the partial area with high viscosity and continues to displace the heavy oil under the thrust of the following agent. Because of the high viscosity of the water-in-oil emulsion and the oil existing as the continuous phase, the effect and characteristic of waterin-oil emulsion flooding are much different from those of the polymeric surfactant agent.

In order to study the oil displacement effect and microscopic mechanism of the two forms of displacement, three 


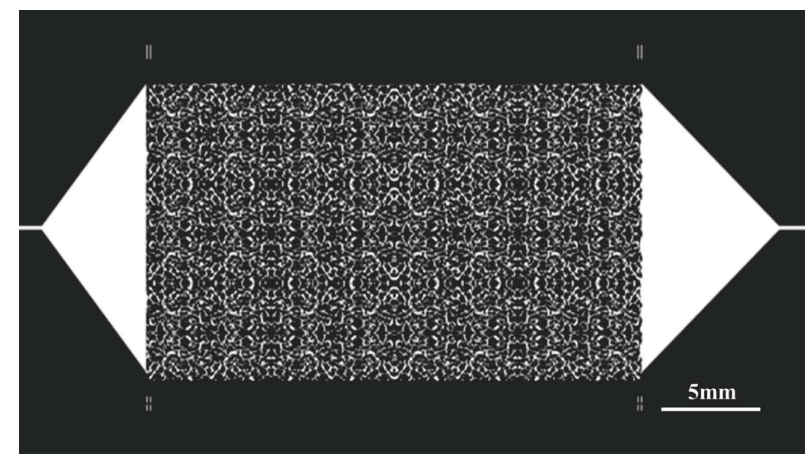

Fig. 2. The schematic diagram of the microstructure.

groups of experiments were carried out in this paper. The first group used deionized water to displace the heavy oil as the control group, and the second group used polymeric surfactant as displacing phase to simulate the first form of displacement. In order to simulate the second form of displacement, the third group carried out the heavy oil displacement experiment with the water-in-oil emulsion as displacing phase, and then used the polymeric surfactant agent to continue the displacement after the end of the water-in-oil emulsion flooding.

The schematic diagram of the microstructure used in the experiment is shown in Fig. 2. The width of the pattern structure in the middle of the model is $1.5 \mathrm{~cm}$, the length is $2.5 \mathrm{~cm}$ and the depth is $30 \mu \mathrm{m}$. In the experiment, heavy oil and displacement phase were injected from one end, and the other end was used as outlet.

\subsection{Experimental procedure}

(1) Study on emulsion phase behavior

The emulsion phase behavior was studied in this paper in order to study the emulsification of polymeric surfactant and heavy oil, and to provide support for the emulsion flooding of the following microfluidic experiments.

Mix the polymeric surfactant with the heavy oil at 1:1 ratio in the test tube and shake the test tube fully. Then leave to rest for several days, and observe the emulsion phase state under a microscope.

(2) Water/polymeric surfactant flooding experiments

The steps of the two groups of experiments (water flooding and polymeric surfactant flooding) are similar. And the experimental steps are as follows:

a) Microfluidic model preparation. The pore throat channels inside the microfluidic model were cleaned with alcohol to remove the impurities on the surface of the model channels, and then dried at $110{ }^{\circ} \mathrm{C}$ for $1 \mathrm{~h}$.

b) Saturating heavy oil. A constant pressure device is used to inject heavy oil into the model under greater pressure until the heavy oil saturates the entire chip.

c) Displacing heavy oil. The microfluidic chip with saturated heavy oil was injected with water or polymeric surfactant at a rate of $0.1 \mu \mathrm{L} / \mathrm{min}$. The whole displacement process was observed under a microscope and the displacement image was collected in real time.

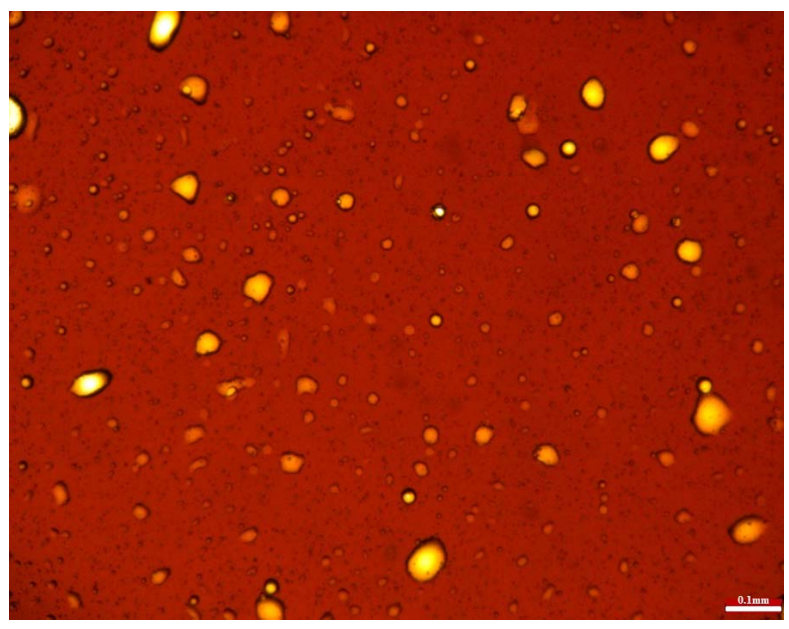

Fig. 3. Water-in-oil emulsion under a microscope.

(3) Water-in-oil emulsion flooding experiment

The steps of this experiment are similar to the first two groups of experiments. After two steps of microfluidic model preparation and saturating heavy oil, the microfluidic chip with saturated heavy oil was first injected with water-in-oil emulsion at a speed of $0.1 \mu \mathrm{L} / \mathrm{min}$, and then injected with polymeric surfactant agent at the same speed after the emulsion broke through the outlet end. The whole displacement process was observed under a microscope and the displacement image was collected in real time.

\section{Results and discussion}

\subsection{Study on emulsion phase behavior}

The emulsion phase state under a microscope is shown in Fig. 3. It can be seen that the heavy oil (in the brown areas) in the emulsion is a continuous phase, while the polymeric surfactant solution (in the white and faint yellow areas) in the emulsion is a dispersed phase. The scale length of the lower right corner of the picture is $0.1 \mathrm{~mm}$ and it can be seen that the diameter of the polymeric surfactant solution droplets in the diagram is less than 100 microns, most of them are about 10 microns and below, which is a typical water-in-oil emulsion.

\subsection{Water/polymeric surfactant flooding experiments}

The microfluidic model was placed horizontally in the oil displacement process. And the process of water flooding experiment is shown in Fig. 4. The microfluidic chip with saturated heavy oil was injected with deionized water at constant speed from the right inlet of the chip. In the picture, the heavy oil is in the brown-yellow region, while the displacement phase is in the transparent region. It can be seen that the fingering is serious and causes water channeling. The process of polymeric surfactant flooding is shown in Fig. 6 . The polymeric surfactant is also injected into the microfluidic chip with saturated heavy oil at constant speed from the right inlet of the chip. It is obvious that the swept area of the poly- 


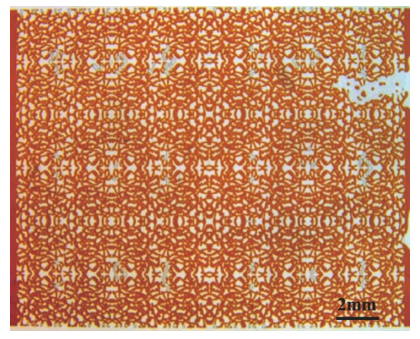

(a)



(b)

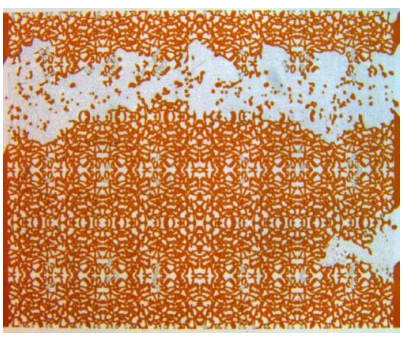

(c)

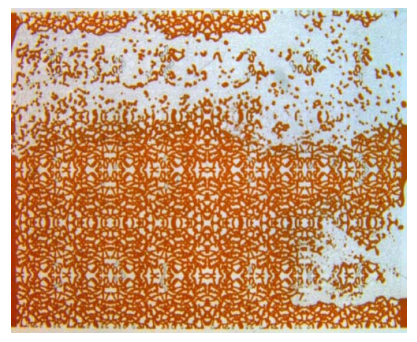

(d)

Fig. 4. Water flooding process.
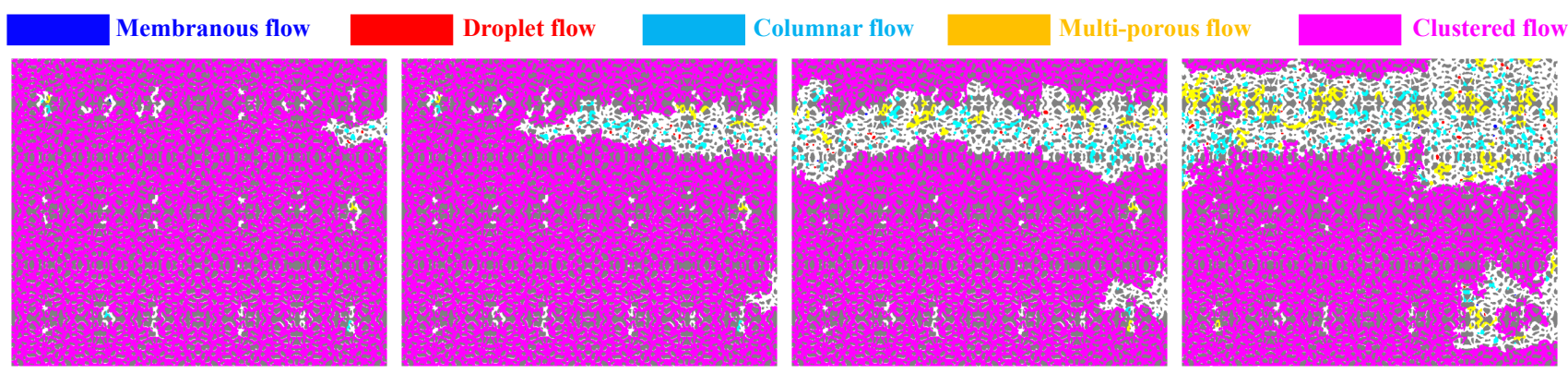

Fig. 5. Classification of remaining oil in water flooding process.

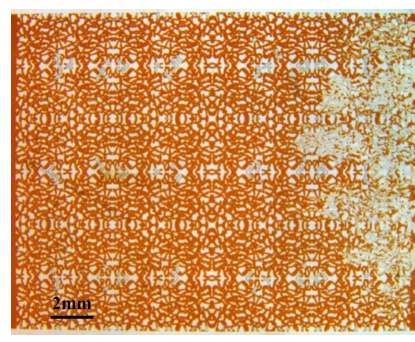

(a)

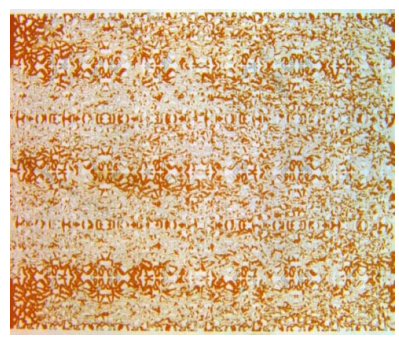

(b)

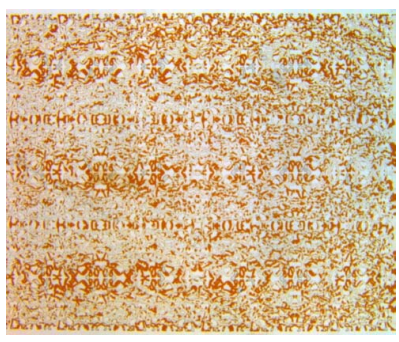

(c)

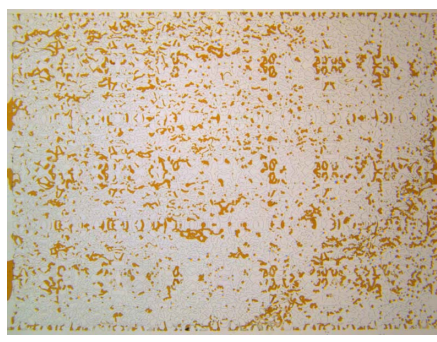

(d)

Fig. 6. Polymeric surfactant flooding process.

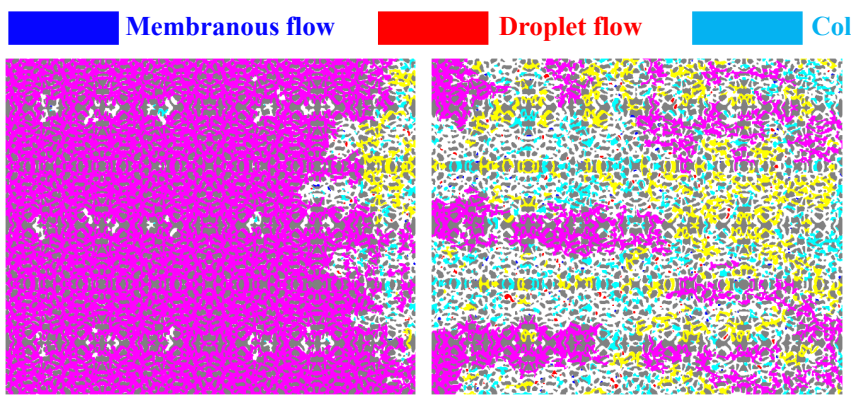

Fig. 7. Classification of remaining oil in polymeric surfactant flooding process. 


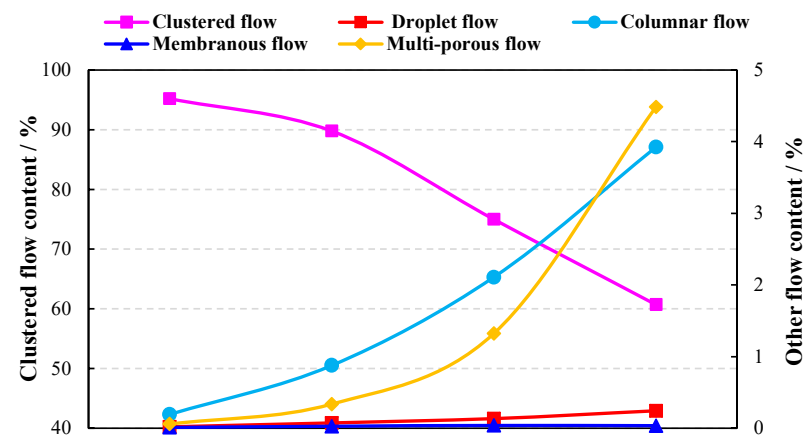

Fig. 8. Content of different types of remaining oil in water flooding process.

meric surfactant flooding is greatly improved compared with the water flooding, and there is no fingering and water channeling phenomenon.

As shown in Fig. 5 and Fig. 7, in order to analyze the type of remaining oil and the effect of displacement, the remaining oil during the displacement process was classified into five types from hard-to-produce to easy-to-produce: Membranous flow (in the blue areas), droplet flow (in the red areas), columnar flow (in the cyan areas), multi-porous flow (in the yellow areas), and clustered flow (purple area), by using Li's classification method (Li et al., 2017, 2018) and related software.

\subsubsection{Experimental result analysis}

As can be seen from Fig. 4 and Fig. 5, the fingering phenomenon is obvious in the process of water flooding and causes uneven displacement front. Therefore, the oil recovery is only $30.6 \%$, and there is still a large amount of unswept remaining oil (clustered flow) in the microfluidic chip after the end of the water flooding. This is because compared with the high viscosity of heavy oil, the viscosity of injected water is too low, which makes mobility ratio too high and make it easy to occur the phenomenon of fingering. When the fingering breaks through the outlet, a large amount of heavy oil is still not affected and extracted, which leads to the small water swept area, low sweep efficiency and low oil recovery. Fig. 8 has shown the changes in the proportion of different types of remaining oil content in Fig. 5 to the initial crude oil content. The left ordinate indicates the clustered flow content while the right ordinate indicates the other remaining oil content. Clustered flow that is easy to produce always maintains a high content ratio while others increase slightly in varying degrees, which means the effect of water flooding is poor.

From Fig. 6 and Fig. 7, it can be seen that the polymeric surfactant flooding has a wide swept area, and there is no obvious fingering phenomenon. The oil recovery is $41.8 \%$ at the time of agent breaking through the outlet, and the final oil recovery reaches $71.7 \%$ at the end of flooding, more than twice the water drive recovery. In terms of data, the remaining oil content is much less than that of water flooding, and the final oil recovery is also higher. In addition, compared with the oil droplet dispersion in Fig. 4 and Fig. 6, the oil droplet driven by polymeric surfactant is more dispersed and the oil

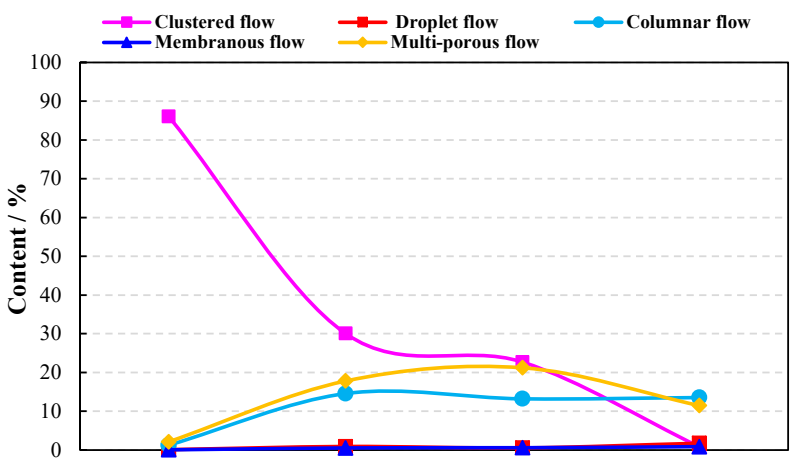

Fig. 9. Content of different types of remaining oil in polymeric surfactant flooding process.

droplet diameter is smaller, which means that the oil flow capacity is higher and it is helpful for the recovery of heavy oil. Fig. 9 has shown the changes in the content of different types of remaining oil in Fig. 7. The contents of the cluster flow that is easy to produce decreased considerably while others increase obviously in varying degrees, which means the effect of polymeric surfactant flooding is much good. In a conclusion, it is obvious that the polymeric surfactant increases the flooding area and oil flow capacity compared with water flooding and plays an important role in enhancing oil recovery.

\subsubsection{Polymeric surfactant flooding mechanism}

According to the analysis of above experimental phenomena, the main polymeric surfactant flooding mechanism is as follows:

(1) Similar to polymer flooding, polymeric surfactant flooding can increase the viscosity of displacement phase, reduce the mobility ratio, avoid the phenomenon of fingering and water channeling, form a more uniform displacement front, expand the range of displacement, and finally improve the efficiency of displacement.

(2) Similar to surfactant flooding, polymeric surfactant flooding can reduce the interfacial tension between oil and water, make it easier for oil phase to disperse and form oil droplets in the water phase, and form stable oil-in-water emulsions to enhance the flow capacity of the oil phase.

(3) In addition, the polymeric surfactant can enter the oil phase in the form of droplets to form the water-in-oil emulsion under diffusion effect. But this effect is not obvious during the displacement process and it will be discussed in detail below.

According to the direct observation of the local oil displacement phenomenon in the microfluidic model in the experiments, the main micro-mechanisms of polymeric surfactant flooding can be obtained as follows:

(1) In the polymeric surfactant flooding process, the typical heavy oil displacement process is shown in Fig. 10.

As can be seen from the Fig. 10, the dispersed oil phase can be dispersed into oil droplets during the displacement process, which can enhance the oil flow capacity. And the oil droplets are difficult to coalesce, which is helpful for the flow and recovery of heavy oil droplets. Comparing the dispersion of heavy oil in the displacement phase in Fig. 4 and Fig. 6, 


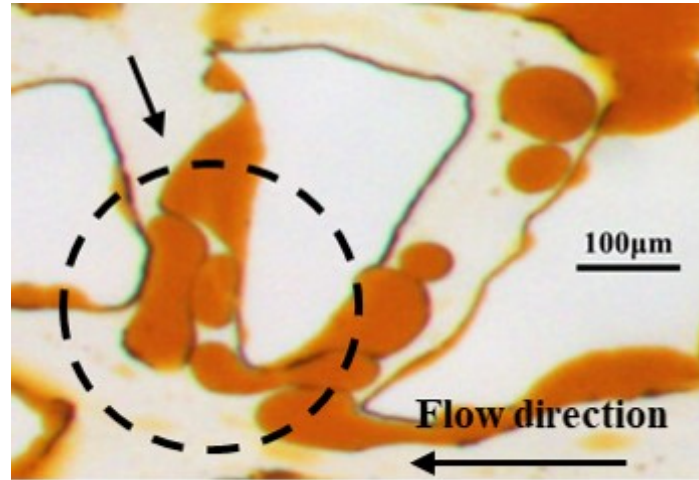

Fig. 10. Micro polymeric surfactant flooding process.

it can be seen that the number of oil droplets dispersed in the polymeric surfactant agent is more than that in the water, and the diameter of the oil droplets is also smaller. These phenomena indicate that in the polymeric surfactant flooding process, the continuous oil phase which is much difficult to flow forms a dispersed phase with stronger flow ability through the shear action of the porous medium and displacing phase, thus becomes easier to flow. And the surfactant characteristics of polymeric surfactant, which can make the agent molecules adsorbed on the surface of the oil phase and reduce the interfacial tension, make the crude oil more easily dispersed in the displacement phase and form the oil phase droplets with smaller diameter. At the same time, the polymeric surfactant agent forms the interfacial membrane on the surface of the oil droplets, which makes it difficult for the oil droplets to coalesce and thus improves the oil phase flow ability to promote the oil phase recovery.

(2) In addition to displacing the heavy oil, the polymeric surfactant could also enter the oil phase in the form of droplets to form the water-in-oil emulsion under diffusion effect, as shown in Fig. 11.

As can be seen from the Fig. 11, the polymeric surfactant enters the oil phase in the form of droplets and thus the waterin-oil emulsion occurs. Compared with the surrounding oil, the color of water-in-oil emulsion becomes obviously darker. In order to study the effect of water-in-oil emulsion on displacing oil, the water-in-oil emulsion flooding experiments are carried out and discussed in detail below.

\subsection{Water-in-oil emulsion flooding}

\subsubsection{Experimental result analysis}

The water-in-oil emulsion flooding process is shown in Fig. 12. After saturating heavy oil, the water-in-oil emulsion is injected into the microfluidic model from the right inlet, and then the subsequent displacement is carried out by injecting polymeric surfactant agent after the end of emulsion flooding. It is obvious in the second picture from left that the swept area of water-in-oil emulsion flooding is particularly wide and it could account for $93.2 \%$ of the model channel. The subsequent polymeric surfactant flooding has also a good displacement effect on water-in-oil emulsion and the recovery at the time
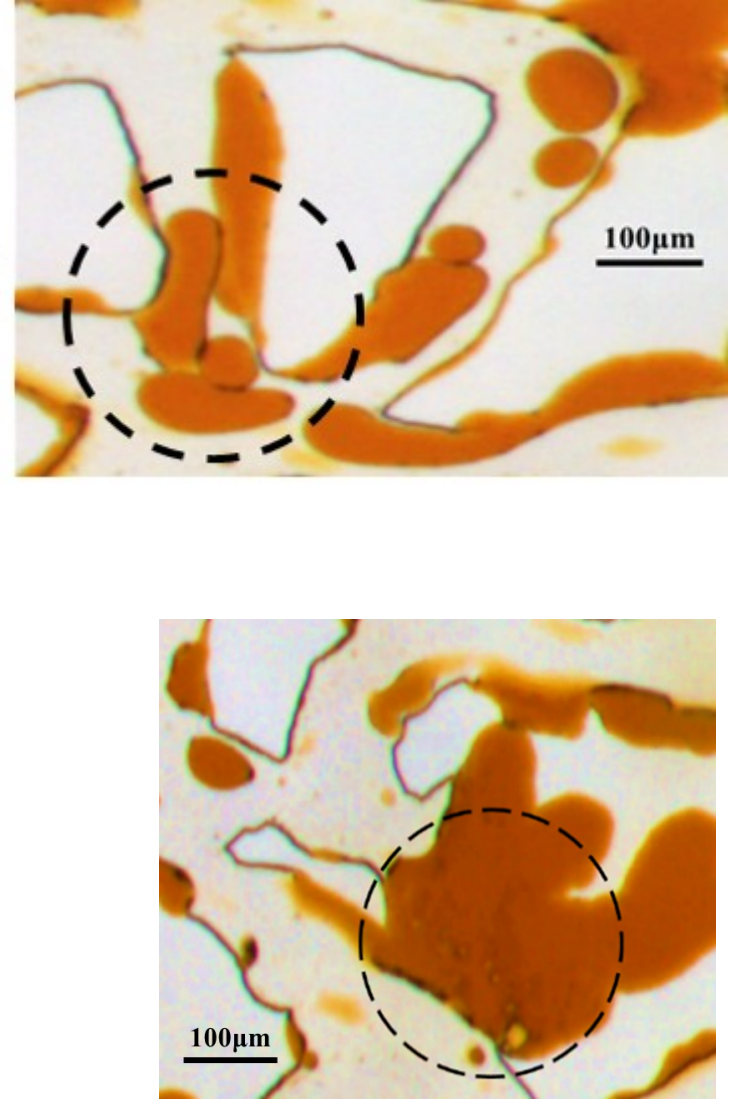

(a)

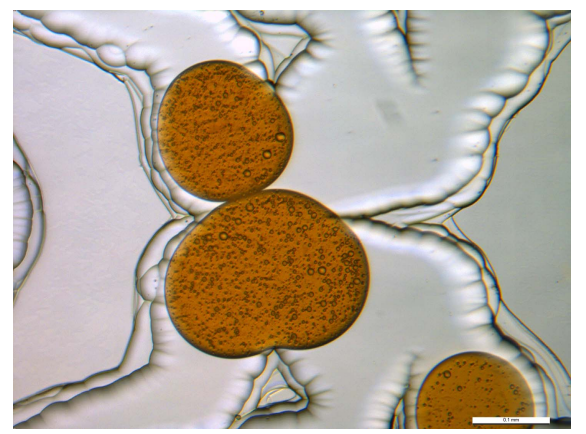

(b)

Fig. 11. The water-in-oil emulsion.

of agent breaking through the outlet is $50.8 \%$ and that at the end of flooding is $81.9 \%$, much more than the recovery of water and polymeric surfactant flooding. Fig. 13 and Fig. 14 have shown the classification and content of remaining oil in agent displacing emulsion process. Cluster flow content drops to zero while others increase obviously in varying degrees, which means the effect of agent displacing emulsion is great. So it can be inferred that compared with water flooding, the water-in-oil emulsion can increase the swept area, avoid the fingering phenomenon, and then help to improve heavy oil recovery. From this point of view, the water-in-oil emulsion flooding with polymeric surfactant flooding followed may be considered as a good EOR method in heavy oil extraction if economic and technical conditions permit. 


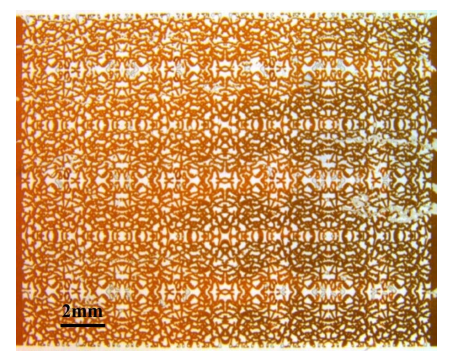

(a)

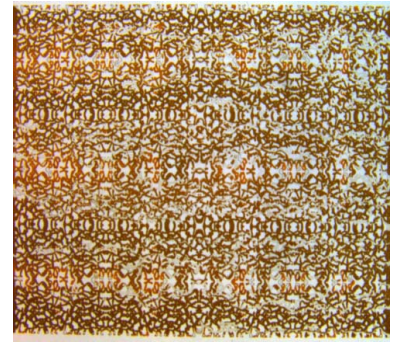

(b)

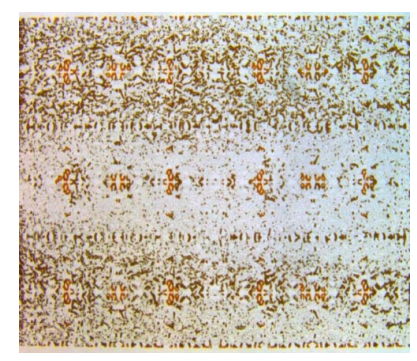

(c)

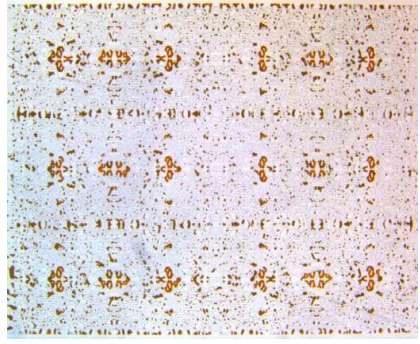

(d)

Fig. 12. Water-in-oil emulsion flooding process: (a) and (b) represent emulsion flooding; (c) and (d) represent agent flooding.
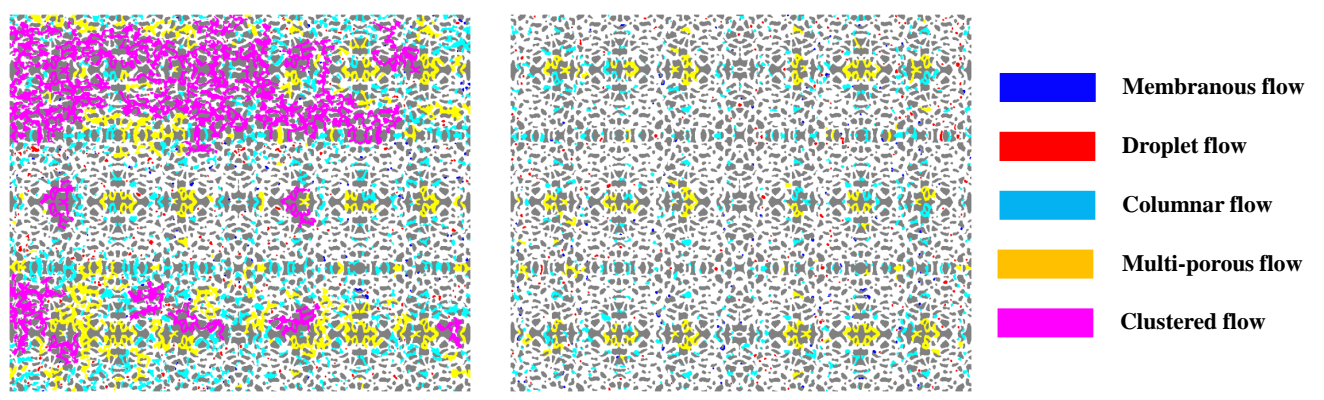

Fig. 13. Classification of remaining oil in agent displacing emulsion process (agent flooding).

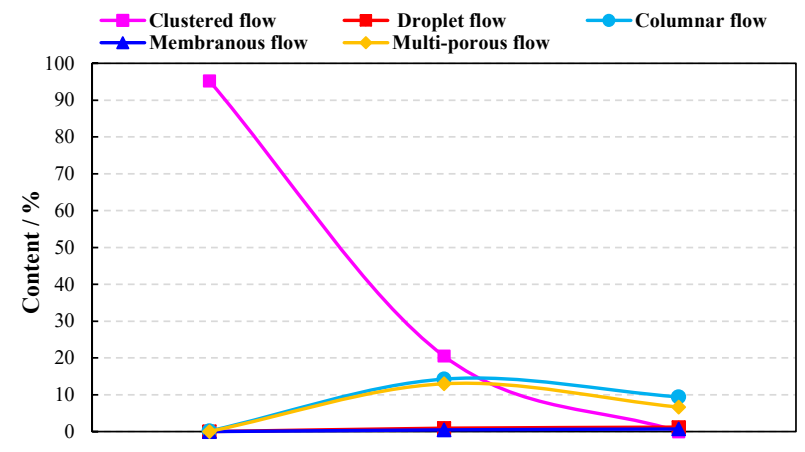

Fig. 14. Content of different types of remaining oil in agent displacing emulsion process.

\subsubsection{Water-in-oil emulsion flooding mechanism}

(1) Compared with the polymeric surfactant agent, the water-in-oil emulsion has a better swept effect, and can enter the dead pore areas which the polymeric surfactant agent cannot affect, as shown in Fig. 15 and Fig. 16.

It can be seen that the water-in-oil emulsion can sweep the dead pores while polymeric surfactant can't. This is because the continuous oil phase in the water-in-oil emulsion can be mixed with the heavy oil in dead pores, like 'miscible flooding', and there is no capillary resistance in the heavy oil displacement process, which makes the polymeric surfactant droplet in the emulsion move into the dead pores and plays the role of oil displacment. Although the polymeric surfactant has the characteristics of surfactant and it can play a role in reducing the interfacial tension, the effect of agent sweeping dead pores is still much less than that of emulsion, which makes its displacement effect on dead pores less obvious.
Besides, the effect of the subsequent polymeric surfactant displacing emulsion in dead pores is not significant, but it has played an important role for the water-in-oil emulsion in enlarging swept area and enhancing heavy oil recovery.

(2) The subsequent polymeric surfactant flooding is carried out after the end of water-in-oil emulsion flooding. During the process of water-in-oil emulsion being driven to the outlet, the polymeric surfactant agent can be adsorbed on the emulsion surface, which greatly reduces the interfacial tension and makes it easy for the water-in-oil emulsion to be cut into smaller diameter emulsion droplets under shearing action and finally form the $\mathrm{W} / \mathrm{O} / \mathrm{W}$ emulsion with strong flow capacity, as shown in Fig. 17. In addition, the polymeric surfactant forms an interfacial film on the emulsion surface, which makes the emulsion droplets difficult to coalesce during the displacement process, and thus improves the flow ability of the emulsion.

\section{Conclusions}

This research investigated the effect of polymeric surfactant in chemical cold flooding for heavy oil by using microfluidic technology. Water, polymeric surfactant and water-in-oil emulsion were used as displacing phase to recovery heavy oil in the microfluidic model. According to the experimental results, the polymeric surfactant flooding has a good EOR effect, and the effect of emulsion flooding is the most ideal, which can sweep the dead pore area that cannot be swept by conventional agents. So the water-in-oil emulsion flooding with polymeric surfactant flooding followed may be considered as a good EOR method in heavy oil extraction if economic and technical conditions permit. 


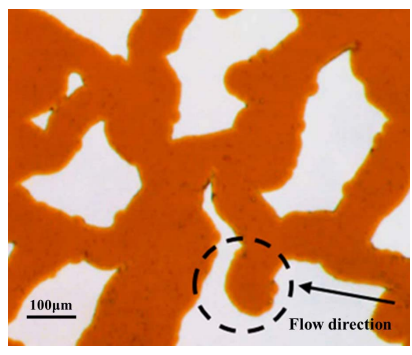

(a)

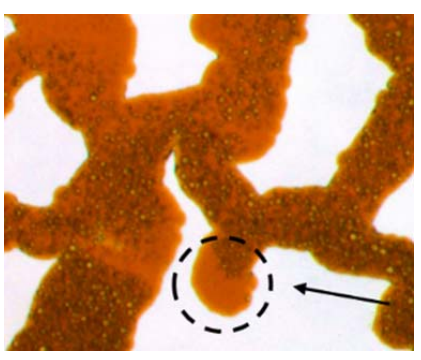

(b)

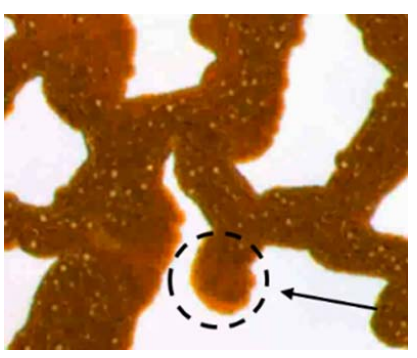

(c)

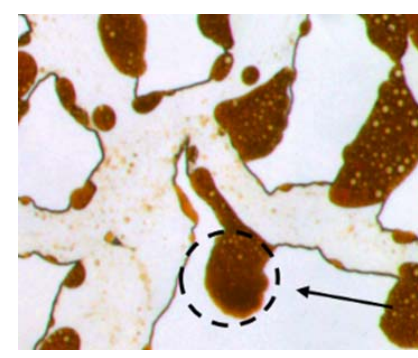

(d)

Fig. 15. Water-in-oil emulsion flooding process: (a) represents saturating oil; (b) represents emulsion flooding 1; (c) represents emulsion flooding 2; (d) represents agent flooding.

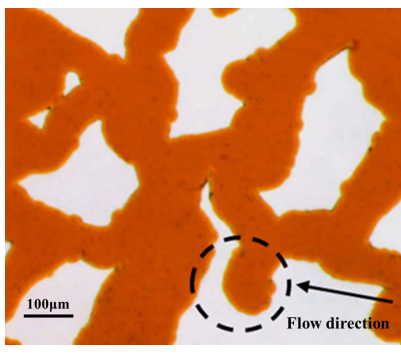

(a)

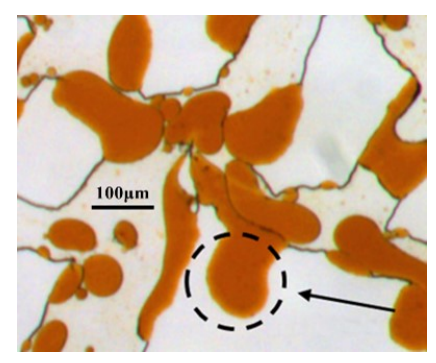

(b)

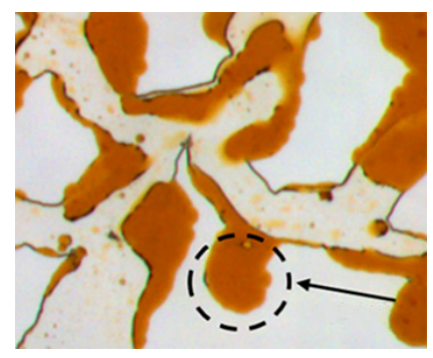

(c)

Fig. 16. Polymeric surfactant agent flooding process: (a) represents saturating oil; (b) represents emulsion flooding 1; (c) represents emulsion flooding 2.

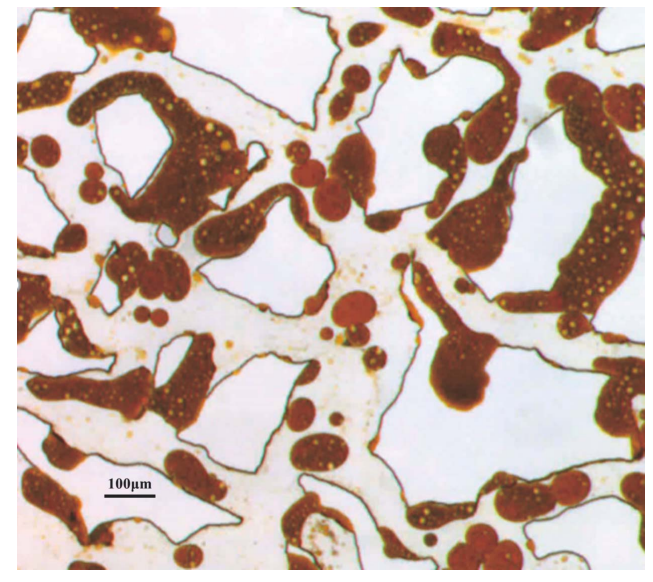

Fig. 17. W/O/W emulsion.

From the whole study, the following conclusions were drawn:

1) The polymeric surfactant flooding has two forms of displacement in chemical cold flooding for heavy oil, one of which is the direct displacement for the heavy oil by the polymeric surfactant and the other is waterin-oil emulsion displacing oil with subsequent polymeric surfactant displacing emulsion.

2) The polymeric surfactant can increase the viscosity of displacement phase and reduce the mobility ratio, which contributes to the enlargement of swept area. And it can reduce the interfacial tension between oil and water, make the oil phase more susceptible to be sheared and form droplets with smaller diameter in the displacement process. Furthermore, the polymeric surfactant agent can form the interfacial film on the oil droplets surface to prevent coalescence, which is helpful to form a more stable water-in-oil emulsion and thus enhances the flow ability of oil phase.

3) The water-in-oil emulsion can be mixed with heavy oil due to its continuous oil phase, which makes it possible to sweep the dead pore area, and thus enhances oil recovery.

\section{Acknowledgement}

This research was supported by State Key Laboratory of Petroleum Resources and Prospecting in China University of Petroleum (Beijing). In addition, we sincerely thank the editor from Advances in Geo-Energy Research for their time and efforts on improving the quality of this paper.

\section{Conflict of interest}

The authors declare no competing interest.

Open Access This article, published at Ausasia Science and Technology Press on behalf of the Division of Porous Flow, Hubei Province Society of Rock Mechanics and Engineering, is distributed under the terms and conditions of the Creative Commons Attribution (CC BY-NC-ND) license, which permits unrestricted use, distribution, and reproduction in any medium, provided the original work is properly cited.

\section{References}

Alvarado, V., Manrique, E. Enhanced oil recovery: An update review. Energies 2010, 3(9): 1529-1575.

Cao, Y., Li, H. Interfacial activity of a novel family of polymeric surfactants. Eur. Polym. J. 2002, 38(7): 1457- 
1463.

Deng, S., Lü, W., Liu, Q., et al. Research on oil displacement mechanism in conglomerate using CT scanning method. Pet. Explor. Dev. 2014, 41(3): 365-370.

Dranchuk, P.M., Scott, J.D., Flock, D.L. Effect of the addition of certain chemicals on oil recovery during water flooding. J. Can. Pet. Technol. 1974, 13(3): 27-36.

Farouq Ali, S.M., Figueroa, J.M., Azuaje, E.A., et al. Recovery of Lloydminster and Morichal crudes by caustic, acid and emulsion floods. J. Can. Pet. Technol. 1979, 18(1): 53-59.

Gbadamosi, A.O., Junin, R., Manan, M.A., et al. An overview of chemical enhanced oil recovery: Recent advances and prospects. Int. Nano Lett. 2019, 9(3): 171-202.

Jamshidi, T., Zeng, F., Tontiwachwuthikul, P. Viability of carbonated water injection (CWI) as a means of secondary oil recovery in heavy oil systems in presence and absence of wormholes: Microfluidic experiments. Fuel 2019, 249: 286-293.

Lee, J., Babadagli, T. Comprehensive methodology for chemicals and nano materials screening for heavy oil recovery using microemulsion characterization. J. Pet. Sci. Eng. 2018, 171: 1099-1112.

$\mathrm{Li}$, L. Performance evaluation of polymer surfactant solution and study on oil displacement effect. Journal of Yangtze University (Natural Science Edition) 2012, 9(8): 74-75. (in Chinese)

Li, J., Jiang, H., Wang, C., et al. Pore-scale investigation of microscopic remaining oil variation characteristics in water-wet sandstone using CT scanning. J. Nat. Gas Sci. Eng. 2017, 48: 36-35.

Li, J., Liu, Y., Gao, Y., et al. Effects of microscopic pore structure heterogeneity on the distribution and morphology of remaining oil. Pet. Explor. Dev. 2018, 45(6): 1112-1122.

Li, J., Su, H., Jiang, H., et al. Application of microfluidic models in the oil and gas field development. Petroleum Science Bulletin 2018, 3(3): 284-301. (in Chinese)

Lifton, V.A. Microfluidics: An enabling screening technology for enhanced oil recovery (EOR). Lab Chip 2016, 16(10): 1777-1796.

Mohammadzadeh, O., Sedaghat, M.H., Kord, S., et al. Porelevel visual analysis of heavy oil recovery using chemicalassisted waterflooding process-Use of a new chemical agent. Fuel 2019, 239: 202-218.

Raffa, P., Broekhuis, A.A., Picchioni, F. Polymeric surfactants for enhanced oil recovery: A review. J. Pet. Sci. Eng. 2016, 145: 723-733.

Shang, X., Bai, Y., Sun, J., et al. Performance and displacement mechanism of a surfactant/compound alkaline flooding system for enhanced oil recovery. Colloid Surf. A 2019, 580: 123679.

Sun, J., Xu, X., Wang, J., et al. Synthesis and emulsification properties of an amphiphilic polymer for enhanced oil recovery. J. Disper. Sci. Technol. 2010, 31(7): 931-935.

Wang, C., Wang, X., Zheng, J., et al. Microscopic visual experiment study of heavy oil gravity drainage. Oilfield Chemistry 2014, 31(1): 79-81. (in Chinese)

Wang, J., Zhu, L., Zhang, F., et al. Microfluidic tools for lipid production and modification: A review. Environ. Sci. Pollut. Res. 2019, 26(35): 35482-35496.

Wu, X., Zhong, C., Lian, X., et al. Solution properties and aggregating structures for a fluorine-containing polymeric surfactant with a poly (ethylene oxide) macro-monomer. Roy. Soc. Open Sci. 2018, 5(8): 180610.

Yan, L. Analysis of characteristics and oil displacement mechanism of new oil displacement agent-polymer surfactant. Journal of Yangtze University (Natural Science Edition) 2014, 11(26): 110-112. (in Chinese)

Yu, F., Jiang, H., Xu, F., et al. A multi-scale experimental study of hydrophobically-modified polyacrylamide flood and surfactant-polymer flood on enhanced heavy oil recovery. J. Pet. Sci. Eng. 2019, 182: 106258.

Zhang, Z., Li, J., Zhou, J. Microscopic roles of "viscoelasticity" in HPMA polymer flooding for EOR. Trans. Porous Media 2011, 86(1): 199-214. 